\title{
EFEITOS DA SUPLEMENTAÇÃO DE ALTO NÍVEL DE COBRE E DE BIOTINA NA DIETA DE SUÍNOS DE 8 A 20 KG
}

\author{
J.F.M. MENTEN'; P.K. KU'; E.R. MILLER ${ }^{2}$
}

RESUMO: Dois experimentos foram conduzidos para investigar a necessidade de suplementação de biotina na dieta de leitões recém desmamados quando o cobre é utilizado como promotor de crescimento. Raçóes com níveis marginais de biotina $(0,10 \mathrm{ppm})$, formuladas à base de trigo e farelo de soja, foram suplementadas ou não com $0,4 \mathrm{ppm}$ de biotina (BIO) e $250 \mathrm{ppm}$ de cobre (COB) e fornecidas a 168 leitoes desmamados com 4 semanas de idade e peso médio de 7,8 kg. Os experimentos tiveram duração de 5 semanas. No experimento 1 houve uma interação BIO x COB $(P<0,05)$ no ganho de peso dos leitóes, com BIO promovendo uma melhora de $10 \%$ na ausência de $\mathrm{COB}$, mas não na presença de $\mathrm{COB}$; $\mathrm{COB}$ resultou em um aumento de $16 \%$ no ganho de peso na ausência de BIO, mas não em sua presença . Também houve uma melhora de $7 \%$ na conversão alimentar dos leitões que receberam $\mathrm{COB}(\mathrm{P}<0,05)$. A mesma tendência foi observada no experimento 2 , e embora a magnitude das diferenças tenha sido semelhante, não houve significância $(P>0,05)$. A suplementação conjunta de COB e BIO não melhorou o desempenho dos animais, em relação a sua suplementação individual.

Descritores: cobre, biotina, leitões desmamados.

\section{EFFECTS OF IIIGII LEVEL OF COPPER AND BIOTIN SUPPLEMENTATION IN THE DIET OF WEANLING PIGS (8 - 20 KG)}

\begin{abstract}
Two trials were carried out to investigate the need for supplemental biotin in the diet of weanling pigs when high dietary copper is used as growth promoter. Wheat - soybean meal based feeds, containing marginal levels of biotin $(.10 \mathrm{ppm})$ were supplemented or not with $.4 \mathrm{ppm}$ biotin (BIO) and $250 \mathrm{ppm}$ copper (COP). The diets were fed to 168 piglets weaned at 4 weeks of age with an average weight of $7.8 \mathrm{~kg}$. The trials were conducted for 5 weeks. In trial 1 there was a BIO x COP interaction $(P<.05)$ for weight gain; BIO resulted in a $10 \%$ improvement in gain in absence of COP, but not when COP was present; COP resulted in a $16 \%$ improvement in absence of BIO and no improvement when BIO was present. There was also a main effect of COP on feed efficiency ( $P<.05$ ), resulting in a 7\% improvement. A similar tendency was observed in trial 2 , but the differences were not significant $(P>.05)$. The combination of supplemental BIO and COP did not result in further improvement in performance compared to the individual supplementation.
\end{abstract}

Key Words: copper, biotin, weanling pigs.

\section{INTRODUÇÃo}

A suplementação de cobre em níveis de 150 a $250 \mathrm{ppm}$ na dieta de suínos em crescimento, especialmente no período imediatamente após o desmame, resulta em melhor ganho de peso e conversão alimentar dos animais (MENTEN, 1988). O cobre é usualmente utilizado na forma de sulfato $\left(\mathrm{CuSO}_{4} .5 \mathrm{H}_{2} \mathrm{O}\right)$, constituindo-se em um promotor de crescimento de custo relativamente baixo.
Biotina é uma vitamina essencial que está presente na maioria dos alimentos em quantidade suficiente para atender à exigência de suínos em crescimento, que é estimada $\mathrm{em} 0,05 \mathrm{mg} / \mathrm{kg}$ de ração (NRC, 1988). Entretanto, a biodisponibilidade da biotina contida nos alimentos é muito variável, havendo estimativas tão baixas como $6 \%$ no trigo até niveis superiores a $100 \%$ no milho (ANDERSON et alii, 1978). Uma vez que ocorre síntese de biotina no intestino grosso de suínos (SAUER et alii, 1986), valores elevados de biodisponibilidade podem resultar

\footnotetext{
' Departamento de Zootecnia da E.S.A. "Luiz de Queiroz", Universidade de São Paulo, 13418-900, PIRACICABA,SP.

${ }^{2}$ Department of Animal Science, Michigan State University, East Lansing,MI - 48824 - USA.
}

Sci. agr., Piracicaba, 49(1):163-166, 1992 
da absorção de biotina dos alimentos bem como da biotina produzida pela microflora intestinal.

Existem muitas indicações na literatura de que níveis elevados de cobre na dieta acarretam alterações na flora intestinal, reduzindo sua atividade (MENTEN,1988). BROOKS et alii (1984) surgiram que essa alteração pode levar a uma redução na síntese de biotina, pois esses autores observaram um melhor desempenho de suínos em crescimento quando uma ração contendo $200 \mathrm{ppm}$ de cobre foi suplementada com $0,5 \mathrm{ppm}$ de biotina. Para testar a hipótese de que a suplementação de biotina é necessaria para que o cobre funcione como promotor de crescimento quando os níveis dietéticos dessa vitamina são marginais, dois experimentos foram conduzidos com leitões recémdesmamados.

\section{MATERIAL E METODOS}

A composição das dietas controle utilizadas nos experimentos 1 e 2 encontra-se na Tabela 1. Pelo fato da disponibilidade de biotina no trigo ser baixa, este cereal foi escolhido para o preparo de dietas com nível marginal de biotina. No experimento 2 houve inclusão de óleo de milho nas dietas em razão da hipótese de que a presença de ácidos graxos insaturados pudesse causar uma redução na disponibilidade de biotina aos animais.

Os tratamentos consistiram de dois níveis de cobre (COB): 0 e $250 \mathrm{ppm}$ e dois níveis de biotina (BIO):0 e $0,4 \mathrm{ppm}$ suplementar, num arranjo fatorial, adicionados às dietas controle em substituição a igual quantidade de trigo.

No experimento 1 foram utilizados 96 leitões machos e fêmeas desmamados com quatro semanas de idade e transferidos para uma sala de creche três dias mais tarde. Os animais foram distribuídos de maneira uniforme a 12 baias de acordo com ninhada de origem e sexo. Os quatro tratamentos foram designados ao acaso às baias, com três repetições. Cada baia com oito leitões constituiu a unidade experimental. $O$ peso médio inicial foi de $7,8 \mathrm{~kg}$ e o peso médio final dos animais foi $20,7 \mathrm{~kg}$.

No experimento 2, 72 leitões foram desmamados com 4 semanas de idade e transferidos imediatamente para as mesmas instalações do experimento 1. De acordo com o peso inicial constituiram-se blocos e em cada bloco os animais foram alocados a quatro baias segundo a ninhada de origem e sexo. A unidade experimental consistiu de uma baia com seis leitões. Os pesos médios inicial e final foram, respectivamente, $7,8 \mathrm{~kg}$ e 19,3 kg.
Em ambos experimentos os animais foram mantidos em ambiente climaticamente controlado em baias com piso parcialmente ripado, com uma área de $2,2 \mathrm{~m}^{2}$. Durante o período experimental de 5 semanas os leitões receberam água e ração "ad libitum". Os resultados de ganho díário de peso, consumo diário de ração e conversão alimentar foram submetidos à análise da variância de acordo com GILL(1978).

Tabela 1. Composição percentual das dietas controle nos experimentos 1 e 2.

\begin{tabular}{||lcc||}
\hline Ingredientes & Exp. 1 & Exp. 2 \\
\hline Trigo & 73,9 & 67,8 \\
Farelo de soja & 22,0 & 23,0 \\
Óleo de milho & - & 5,0 \\
Fosfato mono-bicálcico & 1,35 & 1,40 \\
Carbonato de cálcio & 1,20 & 1,20 \\
Sal & 0,30 & 0,35 \\
Premix vitamínico- & & \\
mineral & 0,50 & 0,50 \\
Premix vit. E - Se & 0,50 & 0,50 \\
L - Lisina H Cl & 0,25 & 0,25 \\
Valores Calculados & & \\
Proteína bruta, \% & 17,1 & 16,9 \\
Lisina, \% & 1,03 & 1,04 \\
Cálcio, \% & 0,80 & 0,80 \\
Fósforo, \% & 0,65 & 0,65 \\
Biotina, ppm & 0,10 & 0,10 \\
\hline
\end{tabular}

- Fornece as seguintes quantidades por kg de ração: vit.A,3300 UI; vit.D,660 UI; vit.K, 2,2 mg; riboflavina, 3,3 mg; niacina, $18 \mathrm{mg}$; ácido pantotênico, $13 \mathrm{mg}$; colina, $110 \mathrm{mg}$; vit. $\mathrm{B}_{12}, 30 \mathrm{mcg}$; $\mathrm{Zn}$, $75 \mathrm{mg} ; \mathrm{Mn}, 34 \mathrm{mg} ; \mathrm{Fe}, 60 \mathrm{mg}$; Cu, $10 \mathrm{mg} ; \mathrm{I}, 0,5 \mathrm{mg}$.

bornece $0,1 \mathrm{mg}$ Se e 17 UI vit.E por kg de ração. 
Tabela 2. Efeito de cobre (COB) e/ou biotina (BIO) suplementares na dieta sobre o desempenho de leitões desmamados. Experimento 1.

\begin{tabular}{|c|c|c|c|c|c|c|c|c|}
\hline \multirow{2}{*}{$\begin{array}{l}\text { COB ppm } \\
\text { BIO ppm }\end{array}$} & \multicolumn{2}{|c|}{0} & \multicolumn{2}{|c|}{250} & \multirow[b]{2}{*}{$\mathrm{EPM}^{a}$} & \multicolumn{3}{|c|}{$\mathbf{P}$} \\
\hline & 0 & 0,4 & 0 & 0,4 & & $\mathrm{COB}$ & BIO & COB X BIO \\
\hline $\mathrm{N}^{\circ}$ de animais & 21 & 24 & 23 & 24 & & & & \\
\hline GDP, $\mathrm{kg}$ & 0,355 & 0,391 & 0,413 & 0,393 & 0,011 & 0,05 & NS & 0,05 \\
\hline $\mathrm{CDR}, \mathrm{kg}$ & 0,70 & 0,72 & 0,73 & 0,70 & 0,02 & NS & NS & NS \\
\hline $\mathrm{CA}$ & 1,96 & 1,84 & 1,76 & 1,79 & 0,04 & 0,05 & NS & NS \\
\hline
\end{tabular}

GDP - ganho diário de peso

CDR - consumo médio de ração

CA - conversão alimentar

a - erro padrão da média de tratamentos

\section{RESULTADOS E DISCUSSÃO}

Experimento 1. Os resultados de performance dos leitões submetidos aos quatro tratamentos encontram-se na tabela 2. O ganho diário de peso foi afetado pelas dietas, havendo uma interação $(\mathrm{P}<0,05)$ entre os níveis de $\mathrm{COB}$ e de BIO. A suplementação de BIO promoveu um aumento no ganho de peso na ausência de COB suplementar $(P<0,05)$, mas houve uma tendência a redução do ganho de peso na presença de $\mathrm{COB}$. Por outro lado, alto nível de $\mathrm{COB}$ na dieta resultou em maior ganho de peso na ausência de BIO ( $P<0,01)$, mas não quando BIO foi adicionada. $O$ consumo médio diário de ração não foi significativamente afetado pelos tratamentos $(\mathrm{P}>$ 0,05 ). A suplementação de $\mathrm{COB}$, independente do nível de BIO, resultou em melhor conversão alimentar dos leitões $(P<0,05)$.

Experimento 2. Os resultados deste experimento estão apresentados na tabela 3.0 efeito dos fatores $\mathrm{COB}$ e BIO sobre o ganho diário de peso e a conversão alimentar foi da mesma magnitude do observado no experimento 1 , mas apesar disso não foram detectadas diferenças significativas $(P>0,05)$. Isto foi devido à maior variância do erro no presente ensaio. $O$ consumo médio diário de ração também não foi significativamente afetado pelo tratamentos $(P>$ 0,05 ).

No experimento 1 , a suplementação de 250 ppm $C O B$ isoladamente resultou em ganho de peso $16 \%$ maior e em uma melhora de $10 \%$ na conversão alimentar em relação à dieta não suplementada. Estes resultados são consistentes com a média de uma série de estudos com leitões, relatados na literatura (MENTEN, 1988). Por outro lado, a adição de 0,4 ppm BIO isoladamente à dieta controle promoveu uma melhora de $10 \%$ no ganho de peso e de $6 \%$ na conversão alimentar, evidenciando que a ração baseada em trigo e farelo de soja foi marginalmente deficiente em biotina.

As respostas ao $\mathrm{COB}$ ou BIO suplementares obtidas no experimento 2 foram semelhantes às encontradas no experimento 1 . Vantagens de $18 \%$ no ganho de peso e $12 \%$ na conversão alimentar foram obtidas com $250 \mathrm{ppm} \mathrm{COB}$ isoladamente. BIO isoladamente resultou $\mathrm{em}$ um aumento do ganho de peso de $8 \%$. A inclusão de óleo de milho nestas dietas parece não ter causado uma redução na disponibilidade de biotina, pois os resultados obtidos foram similares àqueles quando óleo não foi utilizado.

Em ambos experimentos não houve qualquer evidência de efeito aditivo ou sinérgico quando $\mathrm{COB}$ e BIO foram suplementados simultaneamente.

Em um experimento similar, KORNEGAY et al., (1989) não observaram respostas no desempenho com suplementação de biotina, mas houve uma resposta de $8 \%$ no ganho de peso com a suplementação de 200 ppm COB e $18 \%$ com a suplementação conjunta de 200 ppm COB e 0,44 ppm BIO. Portanto, no caso do referido estudo, em que as dietas eram baseadas $\mathrm{em}$ milho, farelo de soja e soro de leite, a biotina pareceu potenciar a ação do cobre como promotor de crescimento de leitões dos 7 aos $20 \mathrm{~kg}$ de peso. 
Tabela 3. Efeitos de cobre (COB) e/ou biotina (BIO) suplementares na dieta sobre o desempenho de leitões desmamados. Experimento 2.

\begin{tabular}{|c|c|c|c|c|c|}
\hline \multirow{2}{*}{$\begin{array}{l}\mathrm{COB}, \mathrm{ppm} \\
\mathrm{BIO}, \mathrm{ppm}\end{array}$} & \multicolumn{2}{|c|}{0} & \multicolumn{2}{|c|}{250} & \multirow[b]{2}{*}{ EPM $^{*}$} \\
\hline & 0 & 0,4 & 0 & 0,4 & \\
\hline$N^{0}$ de animais & 18 & 18 & 18 & 18 & \\
\hline GPD, $\mathrm{kg}$ & 0,301 & 0,324 & 0,354 & 0,335 & 0,023 \\
\hline CDR, kg & 0,55 & 0,59 & 0,62 & 0,58 & 0,03 \\
\hline $\mathrm{CA}$ & 2,11 & 2,07 & 1,86 & 2,00 & 0,11 \\
\hline
\end{tabular}

GDP - ganho diário de peso

CDR - consumo médio de ração

CA - conversão alimentar

- erro padrão da média de tratamentos

\section{CONCLUSÕES}

1. As dietas controle à base de trigo e farelo de soja usadas neste estudo foram marginalmente deficientes $\mathrm{em}$ biotina.

2. O Cobre foi um promotor de crescimento eficiente quando utilizado isoladamente.

3. Os resultados deste estudo não confirmaram a hipótese de que o uso de alto nível de cobre na dieta de leitões viria a requerer suplementação de biotina, uma vez que a adição de $0,4 \mathrm{ppm}$ BIO à dieta contendo 250 ppm COB suplementar tendeu a piorar o desempenho dos animais.

4. A inclusão de óleo de milho nas dietas não interferiu com os efeitos da suplementação de cobre e biotina.

\section{REFERENCIAS BIBLIOGRÁFICAS}

ANDERSON,P.A.,BAKER,D.H.,MISTRY, S.P. Biossay determination of the biotin content of corn, barley, sorghum and wheat. Journal of Animal Science, Champaign, IL,47: 654-659, 1978.

BROOKS, P.A., MORGAN,D.T.,HASTINGS,K.E. The effect of dietary biotin on the response of growing pigs to copper sulfate used as a growth promoter. In: INTERNATIONAL PIG VETERINARY SOCIETY, 1984. Proceedings, 1984. p.316.
GILL,J.L. Design and analysis of experiments in the animal and medical sciences. vol.1. Ames, IA. lowa. State University Press. $410 \mathrm{p}$.

KORNEGAY, E.T., VAN HEUGTEN, P.H.G., LINDEMANN, M.D., BLODGETT, D.J. Effects of biotin and high copper levels on performance and imune response of weanling pigs. Journal of Animal Science, Champaign, IL, 67: 1471-1477, 1989.

MENTEN, J.F.M. Effects of high dietary copper on the utilization of nutrients and on blood and intestinal variables of starter pigs. East Lansing, MI, 1988. 122p. Tese de Ph.D.

NRC - Nutrient requirements of swine. Washington, DC. National Academy Press, 1988. 93 p.

SAUER, W.C., OZIMEK, L.,DEN HARTOG, L.A., MOSENTHIN, R., VOLKER, L. Biotin synthesis in the larg intestine of the pig. In: ANNUAL FEEDERS DAY REPORT, 1986. University of Alberta, 1986. p.123-124.

Trabalho entregue para publicação em 05.09.91

Trabalho aprovado para publicação em 25.11.91 